\title{
Prediction of Environmental Vibrations Induced by Railway Traffic using a Three-Dimensional Dynamic Finite Element Analysis
}

\author{
G. Kouroussis ${ }^{1}$, L. Van Parys ${ }^{2}$, C. Conti ${ }^{1}$ and O. Verlinden ${ }^{1}$ \\ ${ }^{1}$ Department of Theoretical Mechanics, Dynamics and Vibrations \\ ${ }^{2}$ Department of Civil Engineering \\ Faculty of Engineering, University of Mons (UMONS), Belgium
}

\begin{abstract}
This paper presents the modelling possibilities of a full tridimensional finite element approach for prediction of railway ground-borne vibrations. Non-reflecting boundaries are compared to classical ones, with the emphasis on the modelling of the soil wave propagation and reflection. Considering that the ground wave propagation is a transient problem, the time domain formulation is preferred. Rules about domain dimension and element size are clearly emphasized, in order to establish the best compromise between accuracy and numerical model heaviness. Typical results are presented, considering cases of homogeneous or layered halfspace, and illustrate the calculation of the structural response of a building during the passage of a tram at constant speed.
\end{abstract}

Keywords: railway ground vibrations, finite/infinite element method, viscous boundary, vehicle dynamics, soil-structure interaction, wheel/rail contact, track deflection.

\section{Introduction}

Railway-induced ground-borne vibration has been investigated by many authors over the two last decades, focusing on the propagation of ground vibrations, which have generally been studied with the help of the boundary element method. The last years were fertile in terms of prediction models. Through these models, the influence of the propagation of ground vibration waves is illustrated considering different generation mechanisms (rail deflection, track irregularity, vehicle dynamics, ... ).

The choice of the numerical procedure is made with the concern of an efficient representation of infinity, adding a supplementary difficulty for the modelling. Several theoretical formalisms are suitable to obtain rigorous solutions for soil responses under dynamic load. A numerical model is unavoidable to account for the soil-structure 
interaction problems with great accuracy. The boundary element method (BEM) is based on an integral transformation that makes it possible to reformulate Navier's elastodynamic equations at the surface. The main difficulty is to determine a particular solution (Green's function) considering the specific case of the problem (homogeneous or layered soil, at the surface or under soil load, ...). BEM is numerically efficient but it is practically limited to linear formulations and simple geometries. It also imposes some restrictions for complex structures but some hypotheses can nonetheless be made, neglecting for example the soil-structure interaction under some conditions [1].

The finite element method (FEM) is by contrast able to model a soil with complex geometries, insofar as proper conditions are applied on the domain border. Another advantage is that FEM software is well adapted to non-linear problems. These nonreflecting conditions have recently been emphasized by Wang et al. [2] as elementary boundaries, local boundaries or consistent boundaries. The latter include the infinite element formulation, which is widely used in acoustics and is being proposed more and more by commercial finite element software's. In the case of the railway, the FEM formulation was usually restricted to bi-dimensional analysis (as proposed by Yang et al. [3] or by Yerli et al. [4]) for limiting computational resources, but recently 3-D models have been found in the literature. Kouroussis and his coworkers [5, 6] have proposed a tri-dimensional model using the well-known Lysmer viscous boundary [7] combined to infinite element formulation, in order to estimate the level of vibrations transmitted to the neighbourhood.

Presently, despite the importance of the vehicle dynamics, information on railway traffic vibrations is most often limited to track/soil configuration. This information makes it possible to understand the soil behaviour but cannot be used by the train constructors to verify the influence of some vehicle components. Moreover the eventual coupling between all the subsystems is neglected in the analyses. Kouroussis et al. [5] have presented a study of vehicle/track influence as relevant as possible with the aid of a compound vehicle/track/soil model. Special attention was paid at the excitation mechanism, considering the track and the vehicle behaviour on the forces transmitted to the soil. The vehicle dynamics was investigated and the results have shown that, on top of the general considerations, the ground vibration level strongly depends on the vehicle configuration. The study was therefore limited to homogeneous half-space, without analysing the effect of soil parameters. It is however recognised that soil layering has effects on ground wave propagation (reflection and refraction at the layer interfaces) and induces soil surface vibrations that are completely different from those obtained in the case of homogeneous half-space.

In this paper, the use of this finite/infinite element model is discussed. Some properties of non-reflecting boundaries are presented. Viscous boundaries are analysed in order to demonstrate their efficiency on soil modelling and to show the conditions that imply the best wave absorption. Rules in frequency and time domain analysis are also given, before presenting the detailed implementation of the model in the ground vibration prediction. Practical applications are presented, based on the passing of a 
tram. Free field responses are shown, as well as the structural response of a building placed near the track, illustrating the possibility of the proposed methodology and the interest of the FEM in this field.

\section{Some elements in soil dynamics}

The simulation of unbounded domains in numerical methods is a very important topic in dynamic soil-structure interaction and wave propagation problems. Although its mechanical behaviour is essentially dependent on the size of its solid particles and voids, the soil is often considered as a linear material (the shear strain is smaller than $10^{-5}$ in most practical cases).

The frequency range depends on the purpose of the problem. If the dweller's comfort or structure damage safety is of interest, the frequencies until $80 \mathrm{~Hz}$ are important (with a particular attention in the range between 5 and $20 \mathrm{~Hz}$, according to the standard references). Higher frequencies are anyway attenuated by the soil.

When the soil is modelled as an elastic, homogeneous and isotropic medium, the wave field can be expressed as a superposition of plane waves [8], of two types: longitudinal waves (or $P$-waves) where the particle motion exists in the direction of wave propagation, and shear waves ( $S$-waves), moving perpendicularly to their propagation direction. The wave velocities $c_{P}$ and $c_{S}$ are given by

$$
c_{P}=\sqrt{\frac{2 G(1-\nu)}{\rho(1-2 \nu)}}
$$

and

$$
c_{S}=\sqrt{\frac{G}{\rho}}
$$

where $G, \nu$ and $\rho$ are the shear modulus, the Poisson's ratio and the density of the medium, respectively. Along the surface, additional waves, called Rayleigh waves, can be developed in the case of shear waves reflection on the free surface. These waves propagate in the horizontal direction and their amplitude decreases exponentially with the depth (the energy in concentrated up to three times its wavelength). It is known that the $P$-wave is the fastest, with after the $S$-wave, itselves a bit faster than the Rayleigh wave. In term of transmitted energy, it is the opposite: in the case of a vertical point load acting on the soil surface, the Rayleigh wave transports $67 \%$ of the total energy [9].

In the case of layered media, reflection and refraction increase the problem complexity. Each layer can be defined by the body waves but the surface waves in layered media are dispersive. The frequency dependent phase velocities of the different modes of the layered medium are therefore considered, depending on the configuration and the height of each layer. For this configuration, the use of numerical tools becomes necessary. 


\section{On the modelling of an unbounded medium using the finite element method}

The FEM is more adapted than BEMs if non-linear problems, local discontinuities or complex geometries are present. The main difficulty in mimicking an infinite domain like soil in the finite element method is clearly to correctly define the conditions at the model border. Many possibilities exist, some of them being limited by restrictive conditions, but can be classified according to their nature and complexity, as summarized in Table 1.

Classical boundaries (free and fixed conditions) must be used with care because they impose a total reflection at the border of the domain, leading to loss of accuracy. Knowing the Saint-Venant's principle, the domain size can be taken sufficiently large to decrease the effect of the reflected wave.

Local boundaries have been proposed by many authors for improving the absorption efficiency at the boundary. Local or distributed springs or damper elements can be imposed at the border, working normally and/or tangentially. The main difficulty is to find the correct coefficients. The work of Lysmer and Kuhlemeyer [7] is a perfect example and is without any doubt the best-known local boundary in the literature. They have investigated different possibilities for expressing infinitesimal dashpots and have found a boundary condition analytically expressed by

$$
\begin{aligned}
\sigma & =a \rho c_{P} \dot{w} \\
\tau & =b \rho c_{S} \dot{v} .
\end{aligned}
$$

The normal and shear stress $\sigma$ and $\tau$ depend on the normal and tangent velocities $\dot{w}$ and $\dot{v}$ of the boundary. Parameters $a$ and $b$ are dimensionless and can both vary from 0 to $\infty$. In accordance with [7], $a$ and $b$ are chosen equal to 1 ; these values give maximum absorption for compression and shear body waves treatment. These authors also studied the case of Rayleigh waves, important in a free surface, and they demonstrated that parameters $a$ and $b$ also depend on the depth of the soil surface for Rayleigh waves. For example, at the free surface, $a$ is nearly equal to 2 since $b=0$. At a depth of twice the Rayleigh wavelength, both parameters tend to converge to 1 , as for the body waves.

The most promising consistent boundaries are certainly in the infinite element library. Two possibilities exist to implement infinite elements [10]:

- use of decay function, combined with ordinary finite element shape functions,

- mapping element from a finite to an infinite domain.

The idea behind both element types is to obtain a decay aspect in the shape function in the form of $1 / r^{i}(i=0,1,2, \ldots)$. The goal of using these elements is to establish a behaviour that is similar to the infinite domain. 


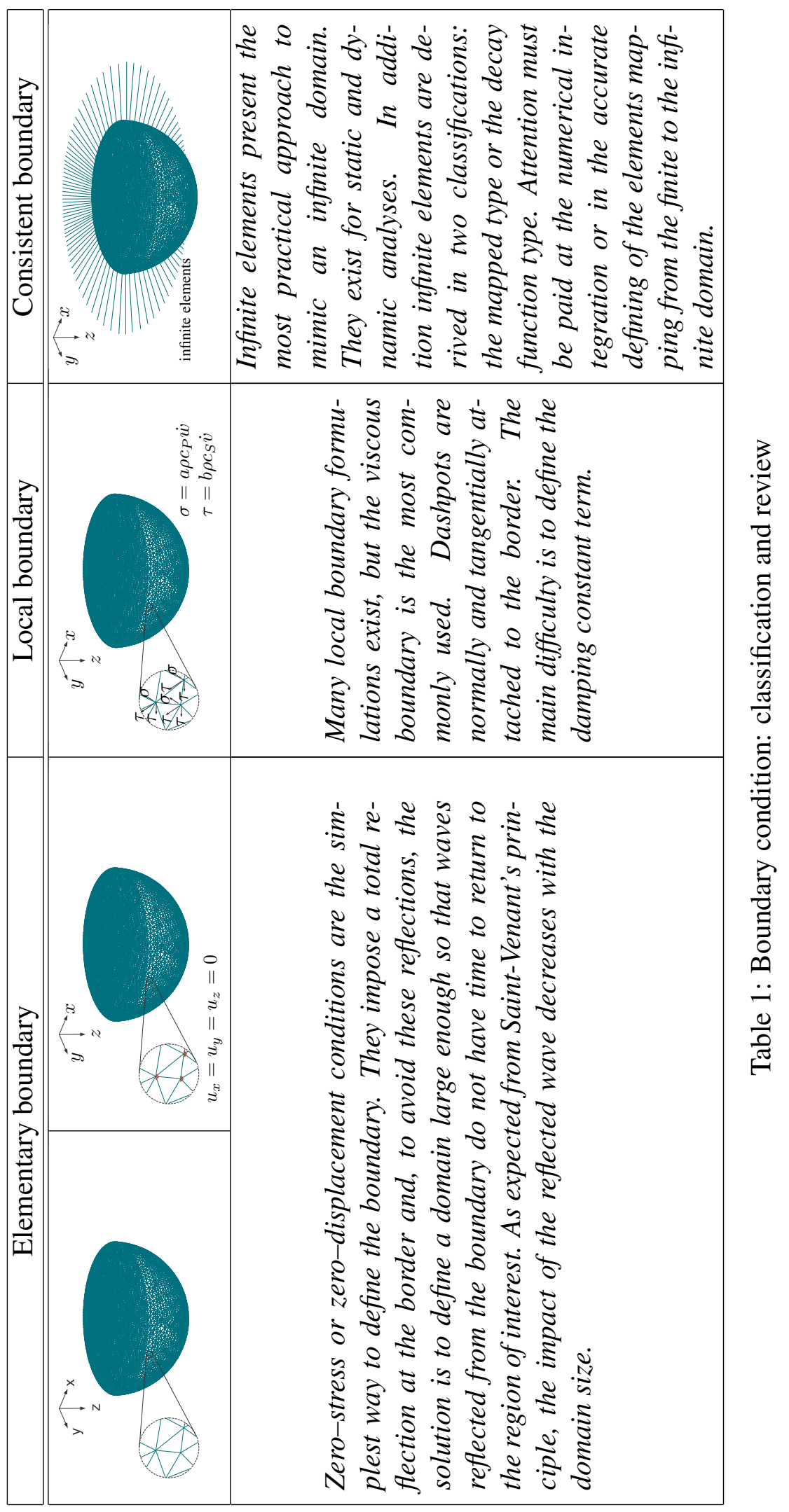




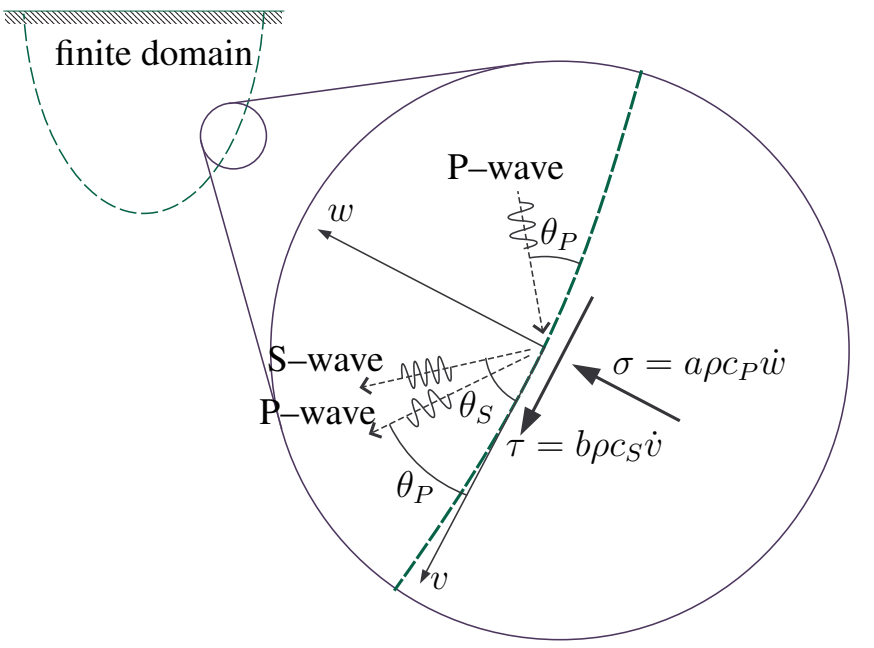

Figure 1: Reflection on the viscous boundary

For example, the mapping procedure is applied to the element in the far field to redefine the infinite domain. The method proposed by Zienkiewicz et al. [11] is certainly the best known, due to its simplicity, and it is still used at the present time. As illustrated in Figure 2(a) (1-D case), node 1, on the interface between finite and infinite elements, at distance $r_{1}=a$ from the pole $r_{0}$ (at $r=0$ ) has the coordinate $\xi=-1$ after mapping. The node 2 coordinate $r_{2}=2 a$ becomes $\xi=0$. The relation between the initial coordinate and the mapped coordinate can be written as

$$
r(\xi)=\frac{-2 \xi}{1-\xi} r_{1}+\frac{1+\xi}{1-\xi} r_{2}
$$

or

$$
r(\xi)=\frac{2 a}{1-\xi}
$$

which gives after inversion

$$
\xi(r)=1-\frac{2 a}{r}
$$

The shape function can finally be combined with standard quadratic interpolation to give

$$
u(\xi)=\frac{1}{2} \xi(\xi-1) u_{1}+\left(1-\xi^{2}\right) u_{2}
$$

where $u_{1}$ and $u_{2}$ are respectively the displacement of nodes 1 and 2 and $u$ the displacements inside the studied element. This function naturally includes a decay aspect, function of $\frac{1}{r}$ and $\frac{1}{r^{2}}$. In fact, using the inverted geometric mapping,

$$
u(r)=\left(-u_{1}+4 u_{2}\right) \frac{a}{r}+\left(2 u_{1}-4 u_{2}\right)\left(\frac{a}{r}\right)^{2}
$$


is found and provides the desired behaviour. In bi- and tridimensional cases, the same procedure is applied for the other coordinates (Figure 2(b)). It is of interest to note that the pole position must be chosen with care to ensure that the element edges in the infinite direction do not cross over each other.

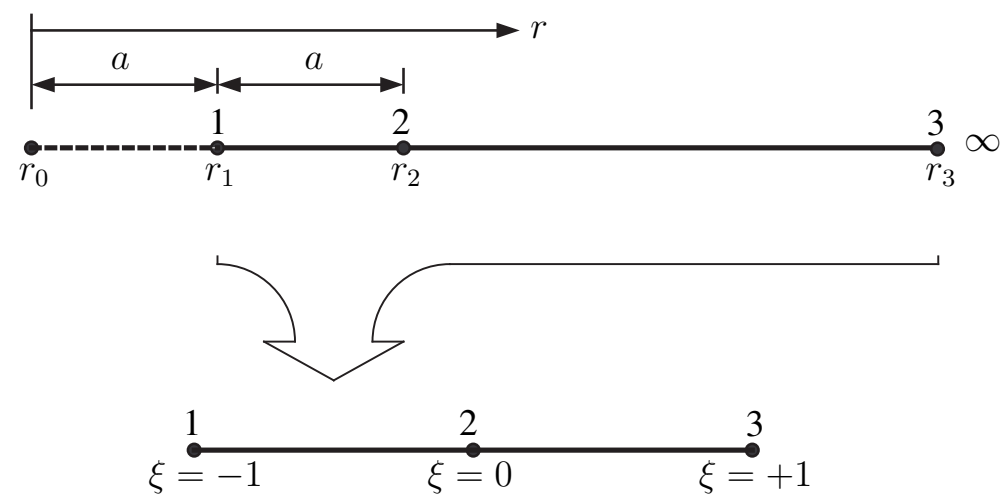

(a) mapped transformation (one infinite dimension)

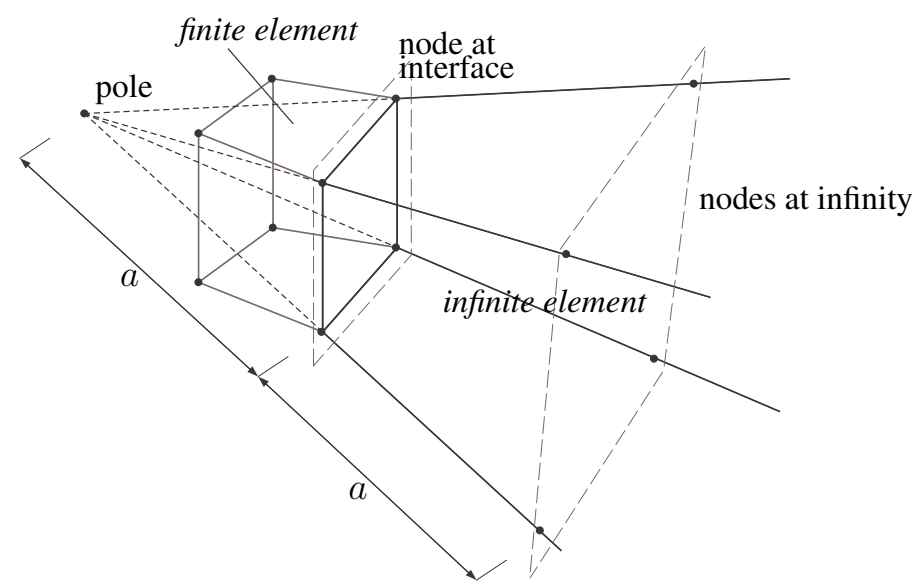

(b) Geometry of the infinite element: position of nodes

Figure 2: Mapped infinite element

\section{Contribution of the viscous boundary and the infi- nite elements}

\subsection{Practical information for finite element software}

To analyse the contribution of local and consistent boundaries, models have been developed under ABAQUS software. Each model consists of a half-sphere composed of finite elements. Specific conditions are applied, such as viscous boundaries according to Eqs. (3) and (4), and mapped infinite elements, connected at the border. Notice that, 
in dynamic analysis under ABAQUS, viscous boundaries are automatically added at the border between finite and infinite elements. Eq. (3) and (4) are used as such, considering $a=b=1$.

The choice of a spherical form allows a convex shape at the border, for insuring the condition of non-crossing infinite elements and for easy meshing. The software can be used to model the soil geometry but an automatic procedure does not exist to add and define the infinite elements at the boundary. A program has been created to fill this deficiency: from the original script file of a FEM model, it reads the geometrical information, searches the boundary nodes and connects the infinite elements with the proper orientation. The use of infinite elements is transparent and perfectly joins the standard finite element procedure.

Classically, rules are used to correctly define a frequency analysis of a general finite/infinite element model. Laghrouche and Le Houédec [12] have analysed various situations, in the case of an harmonic load and in the frequency domain: on top of the classical rule in FE analysis (a minimum of 10 elements per Rayleigh wavelength $\lambda_{R}$ ), the domain size $T_{d}$ must be greater than at least $3 \lambda_{R}$ to minimize the modelling error. In the case of a large frequency band model, these two combined conditions unfortunately impose a large number of elements. Due to the large size of a finite/infinite element model, only the case of a harmonic load (in the frequency domain) was studied and, most often, in the case of 2-D analysis alone [3, 4]. To avoid this limitation, Kouroussis et al. [13] recently studied various situations in frequency and time analyses. As the wave propagation is a transient phenomenon, the domain dimension does not act upon the results if the boundary is defined correctly: a time domain analysis is more appropriate to simulate wave propagation. In the case of tridimensional analysis, computer memory imposes a limited number of elements.

\subsection{Theoretical efficiency of viscous boundary}

Lysmer and Kuhlemeyer [7] have analysed viscous boundary efficiency. For each type of incident body wave, they calculated the ratio between the reflected $E_{r}$ and the incident energy flux $E_{i}$. A mistake unfortunately appears in the final expressions, recently corrected by Kouroussis et al. [14] and summarized as follows.

For an incident primary wave ( $P$-wave) of unitary amplitude and inclined by an angle $\theta$ with respect to the wave front (see Figure 1), the reflection at the viscous boundary develop reflected $P$ and $S$-waves, of amplitude $A_{P}$ and $A_{S}$, respectively. The energy ratio is expressed by

$$
\frac{E_{r}}{E_{i}}=A_{P}^{2}+\frac{\tan \theta_{S}}{\tan \theta_{P}} A_{S}^{2} .
$$

Angle $\theta_{S}$, related to the $S$-waves, is simply defined by

$$
\cos \theta_{S}=\frac{c_{S}}{c_{P}} \cos \theta_{P}
$$

where $c_{P}$ and $c_{S}$ represent the primary and secondary wave velocities, respectively. 
For an incident secondary wave ( $S$-wave), the same observation can be made for the energy flux:

$$
\frac{E_{r}}{E_{i}}=A_{S}^{2}+\frac{\tan \theta_{P}}{\tan \theta_{S}} A_{P}^{2}
$$

available only if $\theta_{S}>\theta_{S, c r}\left(\theta_{S, c r}\right.$ being the critical angle and depending only on Poisson's ratio). In the other case $\left(\theta_{S} \leq \theta_{S, c r}\right)$, Eq. (12) must be substituted by

$$
\frac{E_{r}}{E_{i}}=A_{S}^{2}
$$

Amplitude $A_{P}$ and $A_{S}$ can be calculated by expressing the stress boundary conditions at the interface [7].

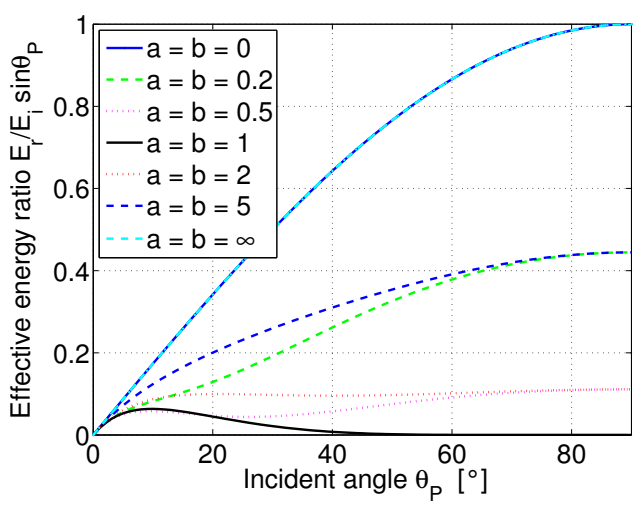

(a) For incident $P$-waves

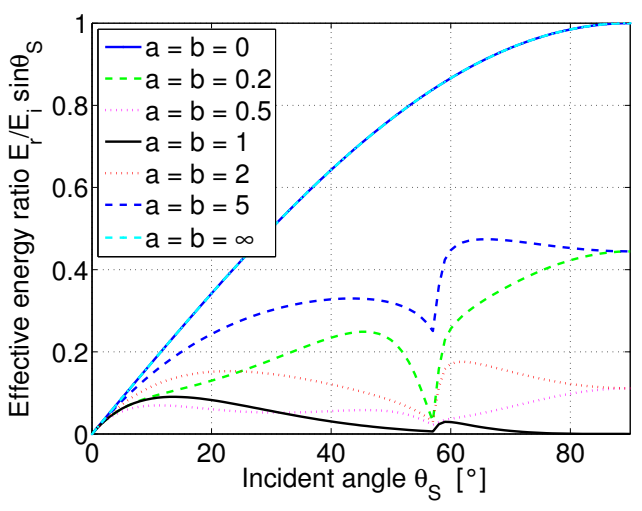

(b) For incident $S$-waves

Figure 3: Effective energy ratio $(\nu=0.3)$

Figure 3 presents the effective energy ratio for two cases, corresponding to the energy ratio multiplied by the sine of incident angle $\left(\theta_{P}\right.$ or $\left.\theta_{S}\right)$, for some values of adimensional constants $a$ and $b$. Particular cases of $a=b=0$ and $a=b=\infty$ correspond to a free and a rigid boundary, respectively. The curves clearly confirm that the choice of unity for value of investigated parameters gives the best absorption. Note that, for a $S$-wave, a discontinuity appears in the curves, at the critical angle $\theta_{S, c r}$. Table 2 summarizes the average of reflected to incident energy, calculated as the ratio between the area under the curve and the total graph area. This reveals that the optimal viscous boundary absorbs nearly four times better than free or rigid boundaries.

\begin{tabular}{|c|c|c|c|c|c|c|c|}
\hline & \multicolumn{7}{|c|}{ value of $a$ and $b$} \\
\cline { 2 - 8 } & 0 & 0.2 & 0.5 & 1 & 2 & 5 & $\infty$ \\
\hline \hline P-wave absorption & $20.6 \%$ & $65.8 \%$ & $91.0 \%$ & $98.3 \%$ & $88.6 \%$ & $62.5 \%$ & $20.6 \%$ \\
$S$-wave absorption & $21.4 \%$ & $70.8 \%$ & $92.4 \%$ & $96.4 \%$ & $85.7 \%$ & $60.5 \%$ & $20.6 \%$ \\
\hline
\end{tabular}

Table 2: Average of reflected to incident energy $(\nu=0.3)$ 


\subsection{The case of an impact load at the soil surface}

A second analysis can emphasize the efficiency of the viscous boundary. The studied case is based on a homogeneous soil with typical dynamic parameters (density $\rho=1600 \mathrm{~kg} / \mathrm{m}^{3}$, Young's modulus $E=120 \mathrm{MN} / \mathrm{m}^{2}$, Poisson's ratio $\nu=0.3$ and viscous damping ratio $\beta=0.0004 \mathrm{~s}$ ). The analysis is based on an impulsive load applied on a $30 \mathrm{~cm} \times 30 \mathrm{~cm}$ square surface. The surface load is defined by the decay function

$$
f(t)= \begin{cases}A_{0} & \text { if } t<t_{0} \\ A_{0}+A e^{\left[-\left(t-t_{0}\right) / t_{d}\right]} & \text { if } t \geq t_{0}\end{cases}
$$

where $A_{0}=0, A=1 \mathrm{~N}, t_{0}=0.05 \mathrm{~s}$ and $t_{d}=0.001 \mathrm{~s}$. These parameters are adjusted in order to get close to an impulsive load. This situation corresponds to a weight falling on the soil surface, as in a test usually performed to determine the soil characteristics. Figure 4 displays the shape of the excitation. It also shows that frequencies up to $100 \mathrm{~Hz}$ are excited with nearly the same proportion.

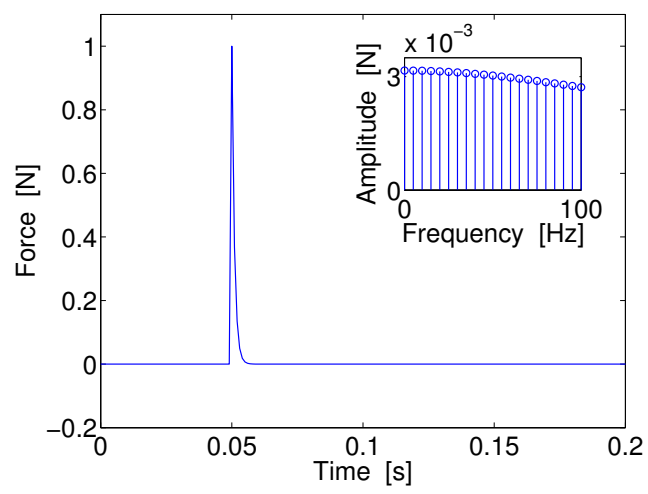

Figure 4: Impact load at the soil surface: excitation characteristics

Figure 5 compares the numerical vertical velocity at the soil surface and at $10 \mathrm{~m}$ from the source, for various viscous boundary configurations (the same value of adimensional constants is used). Two domain sizes $T_{d}=50 \mathrm{~m}$ and $T_{d}=20 \mathrm{~m}$ are studied. Regarding the signals, the following observations can be made:

- The signal is made up of two parts: the incident vibrations (here between 0.05 and $2 \mathrm{~s}$ ), composed partially of the Rayleigh wave contribution, and the reflected signal, more or less significant depending on the values of $a$ and $b$.

- As expected, fixed and free boundaries impose a total reflection at the border. A particular property can be emphasized in these cases: the body waves interaction with the fixed surface develops reflected waves propagating in opposite phase with respect to those generated by the free boundary. This is due to the different impedance at the border. Through the different viscous boundaries, it appears that a good performance is obtained for $a=b=1$, for $T_{d}=50 \mathrm{~m}$ as well as for $T_{d}=20 \mathrm{~m}$. This observation corroborates the small influence of the domain size in time domain analysis if boundaries properly absorb the incident waves. 


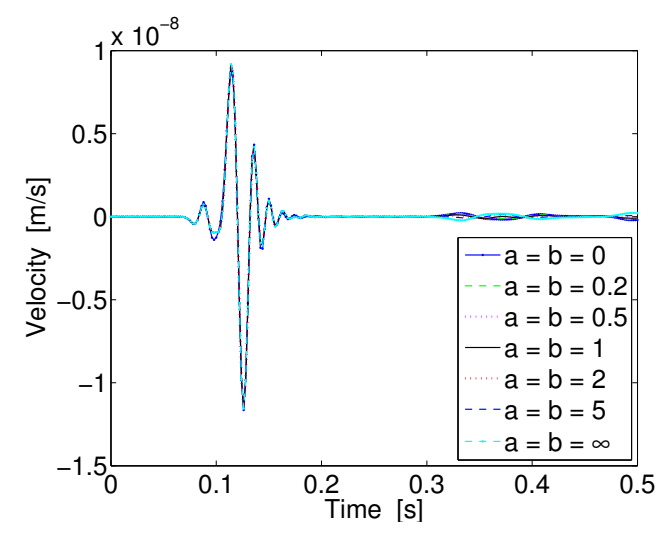

(a) For a domain dimension $T_{d}=50 \mathrm{~m}$

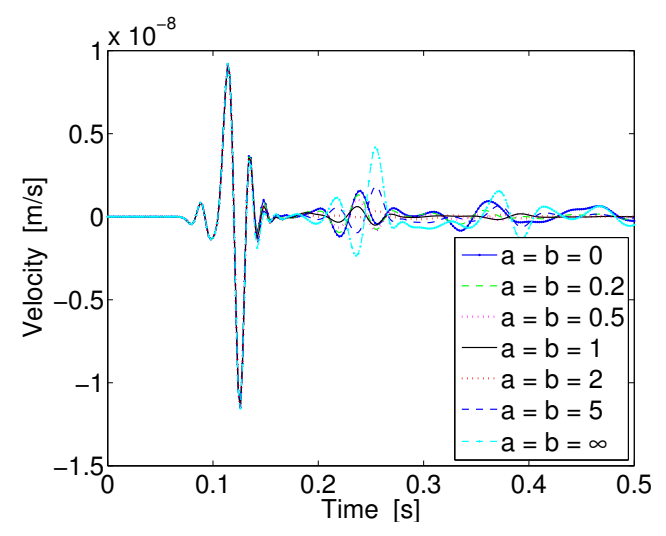

(b) For a domain dimension $T_{d}=20 \mathrm{~m}$

Figure 5: Comparison between various finite element models with a viscous boundary for a homogeneous halfspace: vertical soil surface velocity at $10 \mathrm{~m}$ from the source

- The particular case of $a=b=2$ gives slightly better absorption than the reference case. This can be explained by the fact that the Rayleigh waves, dominating the incident waves, are better absorbed at the soil surface by a viscous boundary with $a=2$. The difference is however negligible.

\subsection{Efficiency with infinite elements}

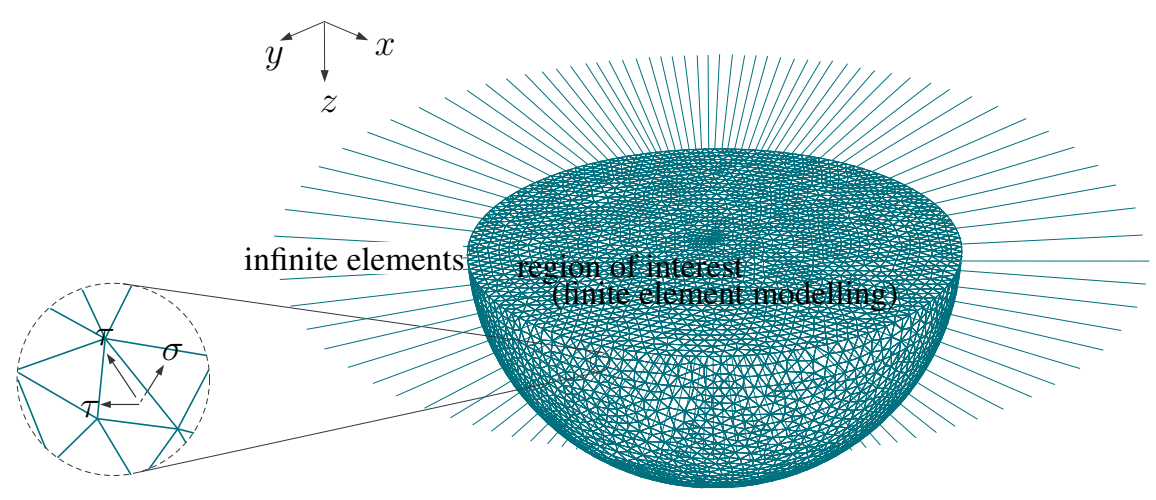

Figure 6: Combined viscous boundary conditions / infinite elements

The commercial ABAQUS software proposes infinite elements in its library, working with the help of the mapping formulation. In static analysis, the infinite elements placed in the domain boundary thereby make it possible to confirm Boussineq's solution. In dynamic analysis, viscous boundaries are automatically added at the border between finite and infinite elements in order to better attenuate the incident waves and to avoid their reflection (Figure 6).

Figure 7 compares the solutions obtained using the combined viscous/infinite elements model. As above, the effect of the domain size is minimal. For both dimensions 


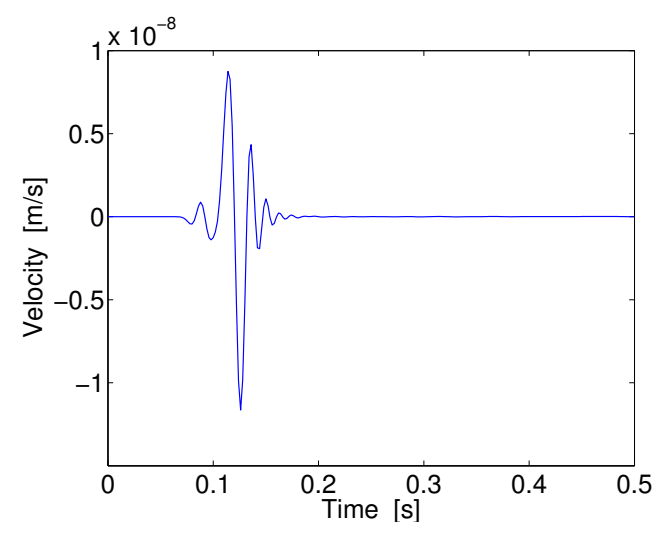

(a) For a domain dimension $T_{d}=50 \mathrm{~m}$

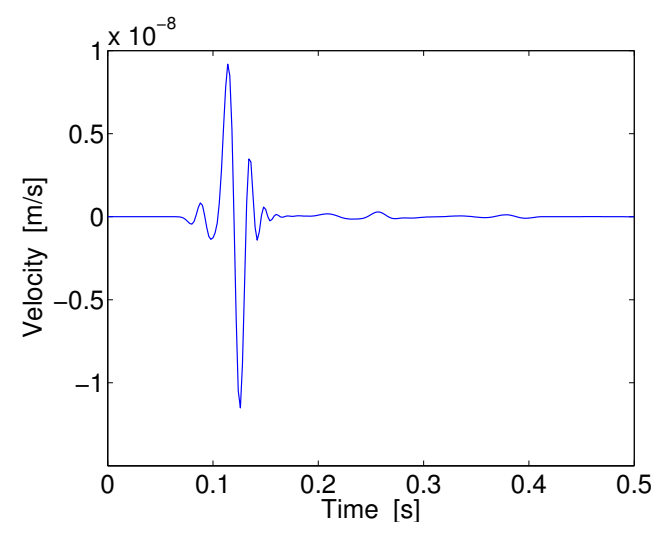

(b) For a domain dimension $T_{d}=20 \mathrm{~m}$

Figure 7: Comparison between various finite element models with combined viscous boundary/infinite elements for a homogeneous halfspace: vertical soil surface velocity at $10 \mathrm{~m}$ from the source

$T_{d}$ of $20 \mathrm{~m}$ and $50 \mathrm{~m}$, practically the same result is obtained. Compared to Figure 5 with the curves with $a=b=1$, a better absorption is obtained, demonstrating the real benefit of the infinite elements. Combined with the viscous boundary, a finite element model offers an interesting tool to predict the dynamic behaviour of soil, particularly if complex geometries or non-linearities are needed.

\section{Calculation of the soil surface forces in the vehi- cle/track subsystem}

A prediction model has been developed, working in two successive steps (Figure 8). The first one is based on the philosophy adopted by the train constructor. The vehicle is modelled using a classical multibody approach. Carbodies and bogie frames are defined as rigid bodies linked by interconnection elements (springs and dampers) representing the suspension. As the vertical motion has a significant effect on ground vibrations, a bidimensional model of the vehicle is sufficient at this stage. It is connected to the track, itself modelled by a classical 2-D and 2-layer model, in accordance with the reviews of Grassie et al. [15] and Knothe and al. [16]. The rail is discretely supported by rigid sleepers. The flexible rail, defined by its Young modulus $E_{r}$, its geometrical inertia $I_{r}$, its section $A_{r}$ and its density $\rho_{r}$, is described by the finite element method. A spacing $L$ of the sleepers has been considered, with a discretization of $N_{n}$ elements for one sleeper spacing. Railpads and ballast are characterized by springs and dampers ( $k_{p}$ and $d_{p}$ for the railpad, $k_{b}$ and $d_{b}$ for the ballast). The sleepers are therefore defined as a lumped mass $m$. The vehicle/track interaction is characterized by the contact law, generally defined as non-linear. The defect on the rail surface is also considered as a local unevenness and/or an overall roughness and can be represented by any kind of deterministic functions. The integration of the established equations of motion is 
performed with the home-made C++ library, EasyDyn, dedicated to second-order differential equations and namely multibody problems $[17,18]$.

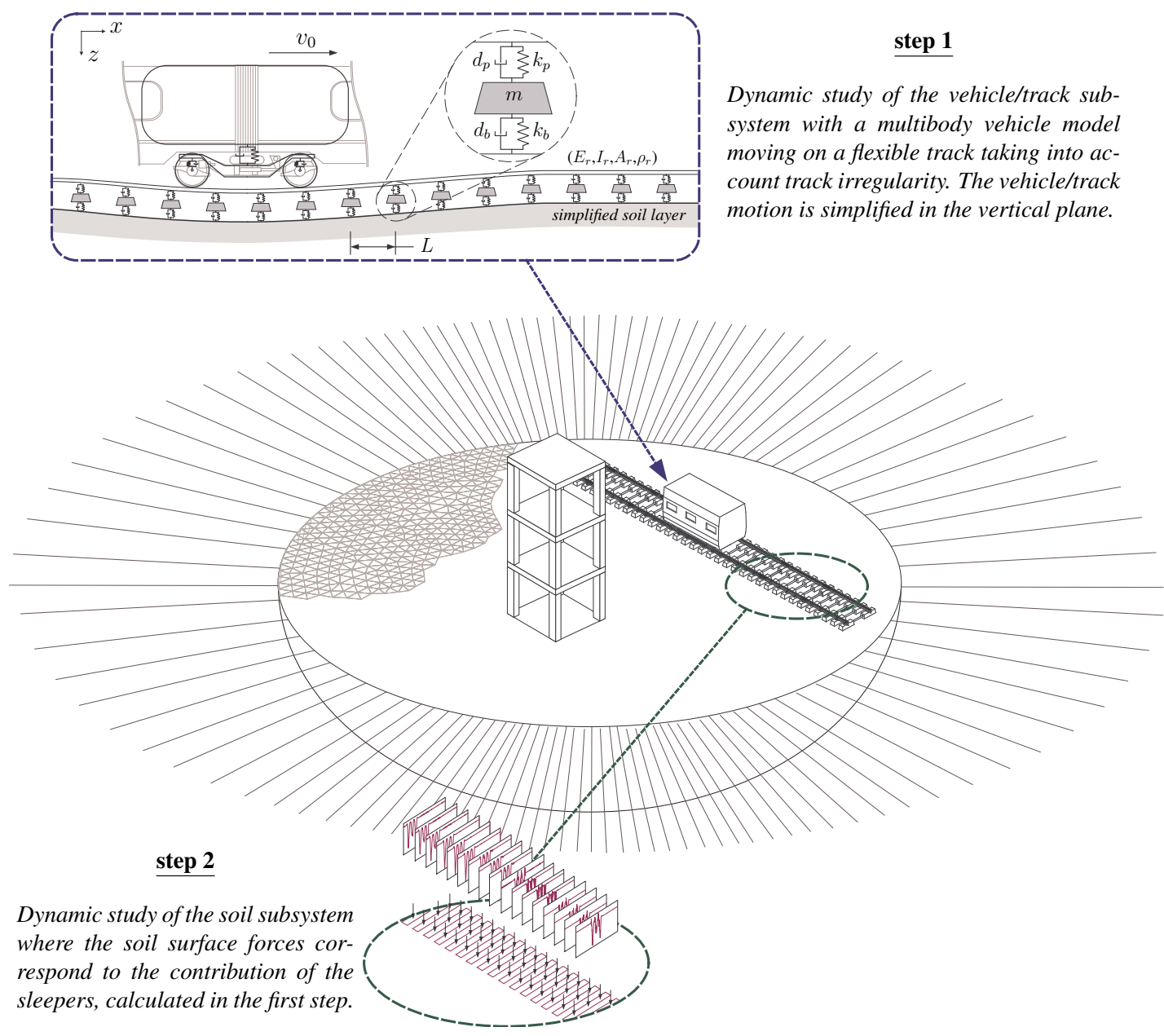

Figure 8: Vehicle/track/soil model, working in two successive steps

The second step considers the reaction forces of the ballast as the loads acting at the surface of the soil. The latter takes all the consideration aspects presented in this paper. The two-step simulation relies on the hypothesis that the track/vehicle and the soil subsystems can be decoupled, which is true only when the soil is stiff enough with respect to the ballast. However, a condensed form of the soil impedance can be included in the track model with the help of a Winkler foundation or more advanced solution [19].

The calculation time required to perform the simulation of the soil dynamics is nevertheless great but can be reduced by using an explicit integration scheme. Figure 9 gives a typical example of calculation time, for implicit or explicit integration schemes, when using 1 or 4 CPU cores. It clearly shows the benefit brought by the explicit integration scheme. Let us mention that the time required to perform the simulation of the soil with the implicit scheme (about 20 days) has been extrapolated. 


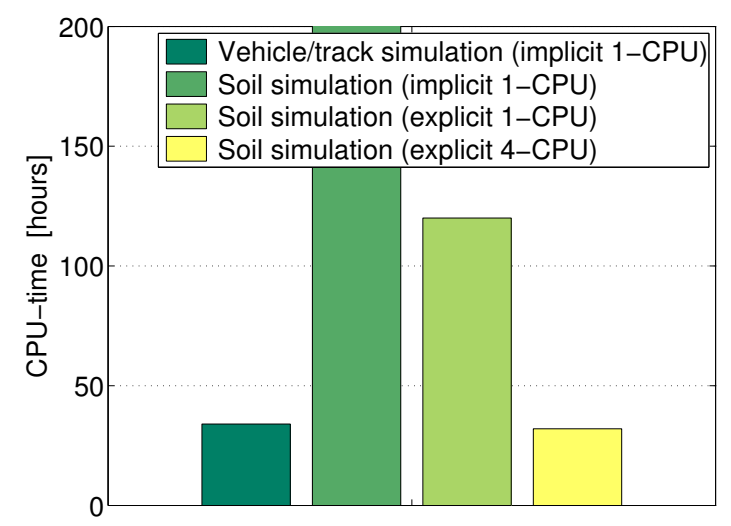

Figure 9: Example of CPU time for vehicle/track and soil simulation (with a QuadCore@2.5GHz PC)

\section{Application to the railway problem}

\subsection{The Brussels T2000 tram}

Due to the low speed, the tram case is a perfect example of the dynamic track interaction where the quasi-static deflection has a small effect on the ground vibrations. Moreover, tramways are very close to buildings and high vibratory nuisances are often felt by the inhabitant. In this context, the tram T2000 LRV of Brussels has been studied in the case of a local defect defined at the rail surface. This multi-car tramway uses independent rotating wheels, resilient motor wheels and a low floor design. It consists of 3 cars for which specific configurations are adopted (Figure 10):

- The central car body has a classical rigid bogie (BR $4 \times 4$ bogie), composed of four independent drive wheels (Figure 10(b)).

- The leading bogies at the extremities are carried by an articulated frame allowing each wheel to be tangent to the rail (BA2000 bogie). It is made up of two independent rotating wheels driven by a motor placed inside the wheels, and two trailer wheels (Figure 10(c)).

Each bogie comprises rubber primary and air-spring secondary suspensions. The main dimensions are given in Figure 10(a).

A multibody model of the vehicle is derived and is defined by 18 degrees of freedom $(d o f)$, to take into account the vehicle bounce and pitch motions. The inputs of the model (urban railway parameters and soil parameters) have been experimentally identified [5] on the tramway site of Haren (Brussels). The soil FIEM dimension is equal to $T_{d}=25 \mathrm{~m}$, which is sufficient for analysing this kind of problem. The track model consists of 532 dof taking into account the flexible rail, the sleepers and a lumped mass model for the foundation. The latter allows to include the soil flexibility directly into the vehicle/track simulation. 


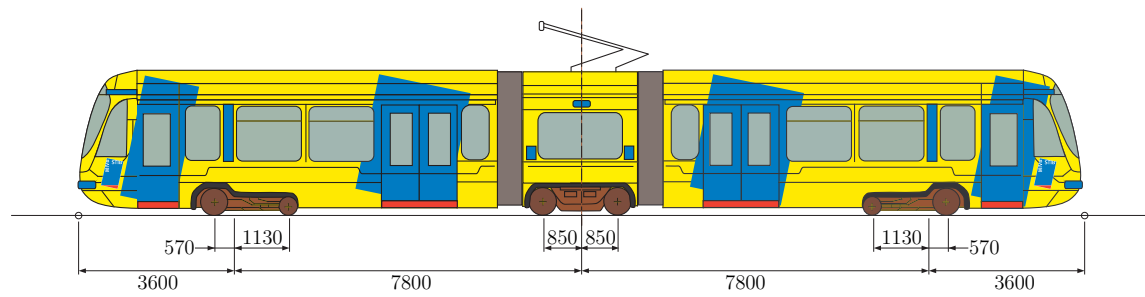

(a) Main dimensions

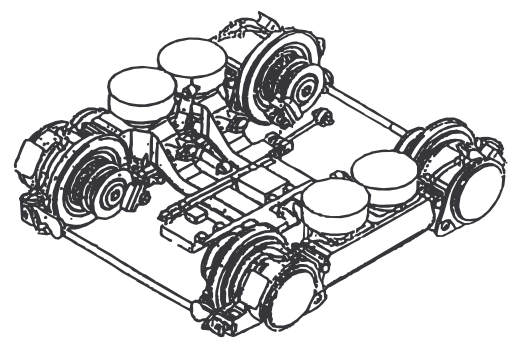

(b) Central bogie BR $4 \times 4$

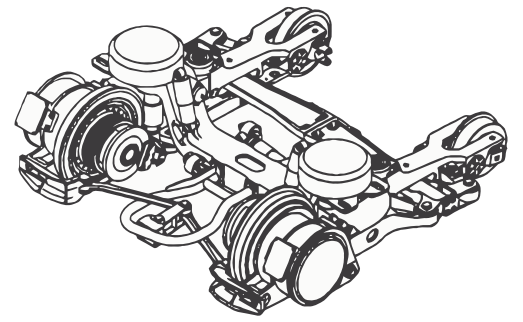

(c) Leading bogie BA2000

Figure 10: Characteristics of the T2000 tram (Bombardier Transport)

\subsection{Free field response during the passage of the tram at constant speed}

The T2000 tramway is simulated in a straight line and the response is calculated at $2 \mathrm{~m}$ from the track, in front of the local rail defect. The desired analysis mainly deals with the interaction of the wheels with the rough rail, to check for the soil surface vertical motion. Two configurations are successively adopted for the soil: a homogeneous soil and a 6-layered soil. Both configurations have been determined using in situ measurements with complementary techniques (refraction survey, SASW). The soil model consists of 568,000 finite/infinite elements and 527,000 dof.

Figure 11 presents the comparison between measurement and model results when the tramway is running at $v_{0}$ of $30 \mathrm{~km} / \mathrm{h}$ and for a distance of $2 \mathrm{~m}$ from the track. Six separate areas are clearly shown in each figure, representing the passing of each wheelset on the defect. It can be seen that the models correctly predict the levels and shapes of the velocity evolution. The layered configuration gives better results than the homogeneous ones, which lightly underestimate the maximum level. This shows that the detailed description of the soil is necessary to accurately predict the ground wave propagation.

\subsection{Structural dynamics of a building near a tramway line}

A building has been modelled by finite elements and placed near the track. The building is representative of classical private houses erected during the $20^{\text {th }}$ century. The house building presents a rectangular shape $(7 \times 10 \mathrm{~m})$ in plane and is composed, in cross section, of a ground floor and an upper one partially involved under the roof. 


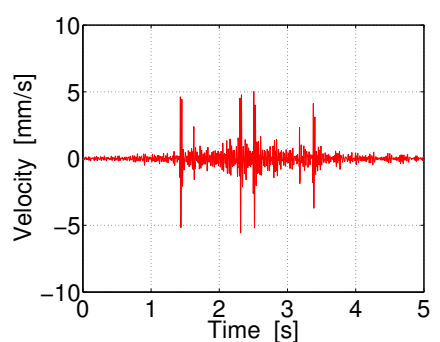

(a) Experimental

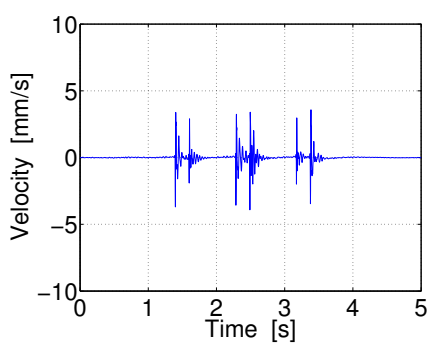

(b) Numerical (homogeneous soil)

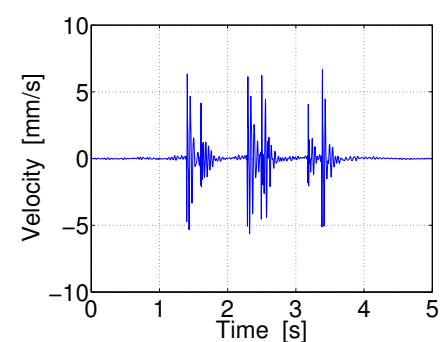

(c) Numerical

(layered soil)

Figure 11: Vertical ground velocity at $2 \mathrm{~m}$ from the track, during the passing of the T2000 tram at speed $v_{0}=30 \mathrm{~km} / \mathrm{h}$

The peripheral masonry walls are composed of plain masonry of non-perforated clay bricks $(0.25 \mathrm{~m}$ thick). The ground floor, the foundation walls and shallow strip foundations are modelled in deep connection with the soil model. The first floor slab is composed of reinforced cast concrete and is embedded inside the masonry walls along each of its sides. The building is close to the track (the front wall is located at $4 \mathrm{~m}$ from the track), corresponding to a realistic situation often encountered in urban area. The layered soil configuration is retained for the simulation.

Figure 12 illustrates the ground wave propagation in the case of free field domain and in the case of the same domain including the studied building. The instants $1.425 \mathrm{~s}$, $2.425 \mathrm{~s}$ and $3.425 \mathrm{~s}$ emphasize the effect of each bogie on this propagation. Maximum and minimum surface velocities are displayed on each sub-figure. The following observations are noteworthy:

- In both situations, the passing of each wheelset induces significant vibrations around the rail defect. In the other cases, the generated ground wave is less important.

- If the vehicle is far from the building, the generation ground wave propagation is identical in both studied cases. When the vehicle is close to the building, the ground waves completely change and the extrema amplitudes are modified. Figure 12(e) and 12(f) show that the presence of the building attenuates the soil surface vibrations in the back of the building and amplifies it in its front.

\section{Conclusion}

The non-reflecting conditions at the border are defined by the combined viscous boundary and infinite elements. Since complex geometries of local non-linear effects are often associated with vibratory nuisance, the time domain simulation is preferred. It also makes it possible to work with reduced model size, without sacrificing the accuracy of the model. Viscous boundary conditions are analysed for efficiency, showing 


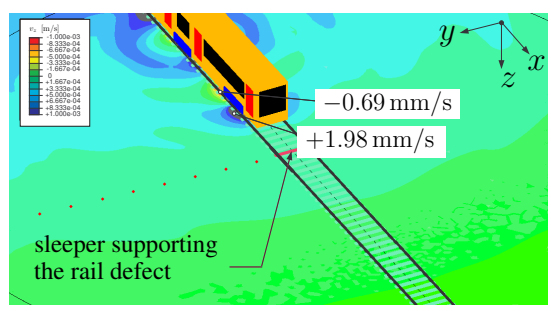

(a) At $0.425 \mathrm{~s}$

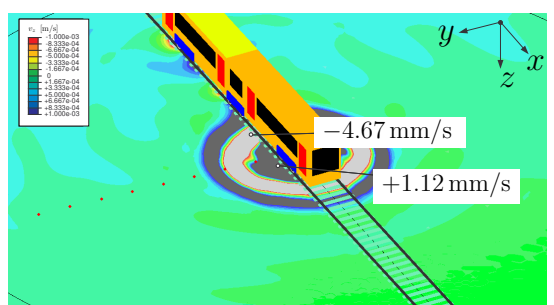

(c) At $1.425 \mathrm{~s}$

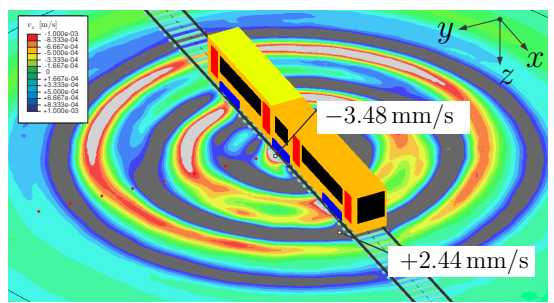

(e) At $2.425 \mathrm{~s}$

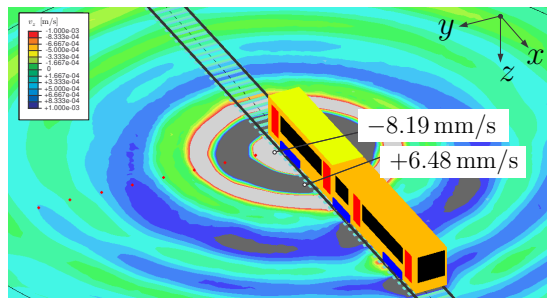

(g) At $3.425 \mathrm{~s}$

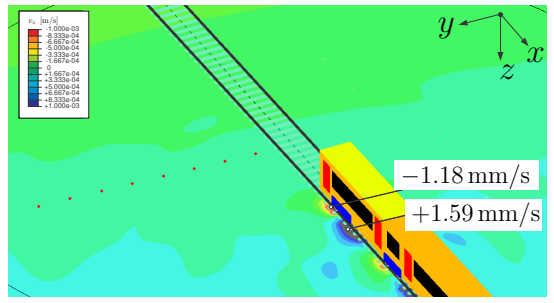

(i) At $4.425 \mathrm{~s}$

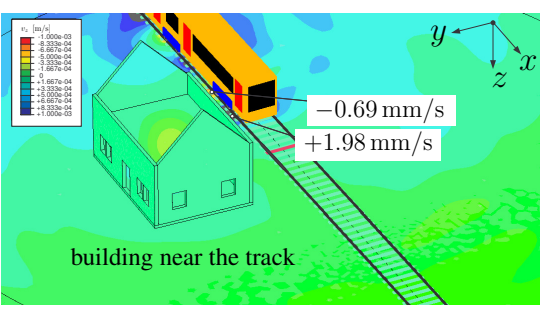

(b) At $0.425 \mathrm{~s}$

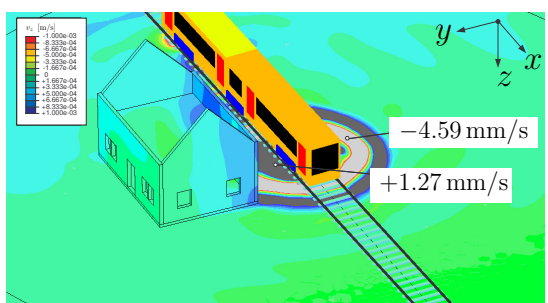

(d) At $1.425 \mathrm{~s}$

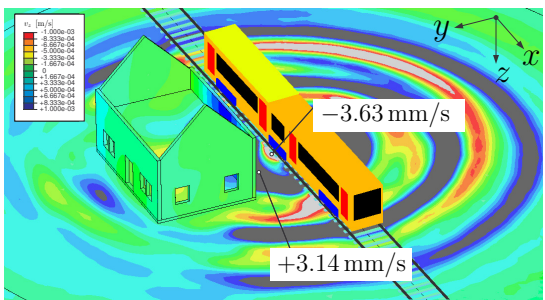

(f) At $2.425 \mathrm{~s}$

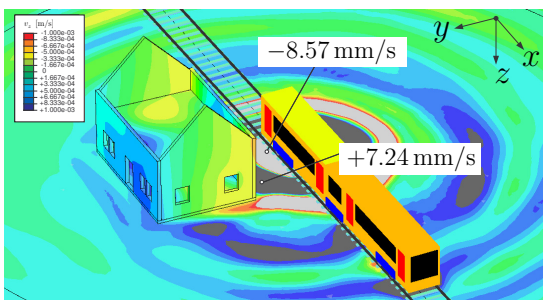

(h) At $3.425 \mathrm{~s}$

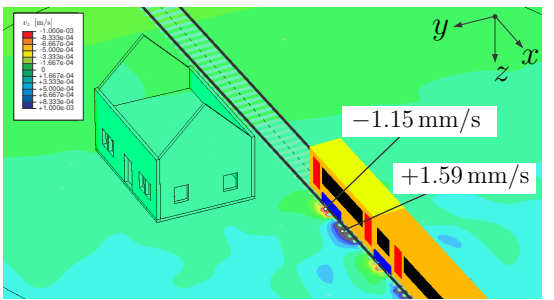

(j) At $4.425 \mathrm{~s}$

Figure 12: Propagation of the free field (left) and structural response (right) vertical component of the soil vibration waves, in the case of a vehicle speed of $30 \mathrm{~km} / \mathrm{h}$ moving along a track with a local defect 
that adimensional parameters intervening in the damping coefficient are adjusted to one for a maximum absorption of body waves.

Applications are presented through a realistic example from the Brussels tramway line. Free field response is displayed and compared to experimental curves, showing good agreement. Structural response is also presented, demonstrating all the possibilities of the finite element method.

\section{References}

[1] S. François, L. Pyl, H.R. Masoumi, G. Degrande, "The influence of dynamic soil-structure interaction on traffic induced vibrations in buildings", Soil Dynamics and Earthquake Engineering, 27(7): 655-674, 2007.

[2] J. Wang, X. Zeng, D.A. Gasparini, "Dynamic response of high-speed rail foundations using linear hysteretic damping and frequency domain substructuring", Soil Dynamics and Earthquake Engineering, 28(4): 258-276, 2008.

[3] Y.B. Yang, H.H. Hung, D.W. Chang, "Train-induced wave propagation in layered soils using finite/infinite element simulation", Soil Dynamics and Earthquake Engineering, 23: 263-278, 2003.

[4] H.R. Yerli, S. Kacin, S. Kocak, "A parallel finite-infinite element model for twodimensional soil-structure interaction problems", Soil Dynamics and Earthquake Engineering, 23: 249-253, 2003.

[5] G. Kouroussis, O. Verlinden, C. Conti, "On the interest of integrating vehicle dynamics for the ground propagation of vibrations: the case of urban railway traffic", Vehicle System Dynamics, 48(12): 1553-1571, 2010.

[6] G. Kouroussis, O. Verlinden, C. Conti, "Free field vibrations caused by highspeed lines: measurement and time domain simulation", Soil Dynamics and Earthquake Engineering, 31(4): 692-707, 2011.

[7] J. Lysmer, R.L. Kuhlemeyer, "Finite dynamic model for infinite media", Journal of the Engineering Mechanics Division, Proceedings of the ASCE, 95(EM4): 859-877, 1969.

[8] T.G. Gutowski, C.L. Dym, "Propagation of ground vibrations: a review", Journal of Sound and Vibration, 49: 179-193, 1976.

[9] G.F. Miller, H. Pursey, "On the partition of energy between elastic waves in a semi-infinite solid", Proceeding of the Royal Society (London), 233: 55-69, 1955.

[10] P. Bettess, Infinite Elements, Penshaw Press, Sunderland (UK), 1992.

[11] O.C. Zienkiewicz, C. Emson, P. Bettess, "A novel boundary infinite element", International Journal for Numerical Methods in Engineering, 19(3): 393-404, 1983.

[12] O. Laghrouche, D. Le Houédec, "Soil-railway interaction for active isolation of traffic vibration", in B.H.V. Topping (Editor), Advances in Simulation and Interaction Techniques, pages 31-36. Civil-Comp Ltd, Edinburgh (Scotland), 1994. doi:10.4203/ccp.23.2.3 
[13] G. Kouroussis, O. Verlinden, C. Conti, "Ground propagation of vibrations from railway vehicles using a finite/infinite-element model of the soil", Proc. IMechE, Part F: J. Rail and Rapid Transit, 223(F4): 405-413, 2009.

[14] G. Kouroussis, O. Verlinden, C. Conti, "Finite dynamic model for infinite media: The corrected solution of viscous boundary efficiency", Journal of Engineering Mechanics, (accepted for publication)(10.1061/(ASCE)EM.19437889.0000250), 2011.

[15] S.L. Grassie, R.W. Gregory, D. Harrison, K.L. Johnson, "The dynamic response of railway track to high frequency vertical excitation", Journal of Mechanical Engineering Science, 24(2): 77-90, 1982.

[16] K. Knothe, S.L. Grassie, "Modelling of railway track and vehicle/track interaction at high frequencies", Vehicle System Dynamics, 22: 209-262, 1993.

[17] O. Verlinden, G. Kouroussis, C. Conti, "EasyDyn: A framework based on free symbolic and numerical tools for teaching multibody systems", in Multibody Dynamics 2005. ECCOMAS Thematic Conference, Madrid (Spain), 2005.

[18] G. Kouroussis, C. Rustin, Q. Bombled, O. Verlinden, "EasyDyn: multibody open-source framework for advanced research purposes", in Multibody Dynamics 2011. Brussels (Belgium), 2011.

[19] G. Kouroussis, G. Gazetas, I. Anastasopoulos, C. Conti, O. Verlinden, "Lumped mass model of vertical dynamic coupling of a railway track on elastic homogeneous or layered halfspace", in 8th European Conference on Structural Dynamics: Eurodyn 2011. Leuven (Belgium), 2011. 